\title{
GENERALIZED MODELLING OF THE STABILIZER LINK AND STATIC SIMULATION USING FEM
}

\author{
COFARU Nicolae Florin \\ professor / Ph.D. Faculty of Engineering/Department Industrial Engineering and Management, 'Lucian \\ Blaga'University, Sibiu, Romania,nicolae.cofaru@ulbsibiu.ro
}

ROMAN Lucian Ion

Ph.D. student, Faculty of Engineering/Department Industrial Engineering and Management, 'Lucian Blaga' University, Sibiu, Romania, roman_lucian_ioan@yahoo.com

OLEKSIK Valentin

professor / Ph.D. Faculty of Engineering/Department Machine and Industrial Equipment, 'Lucian Blaga' University, Sibiu, Romania, valentin.oleksik@ulbsibiu.ro

PASCU Adrian

professor / Ph.D. Faculty of Engineering/Department Machine and Industrial Equipment, 'Lucian Blaga' University, Sibiu, Romania, adrian.pascu@ulbsibiu.ro

\begin{abstract}
This paper proposes an organological approach of one of the components of front suspension, namely anti-roll power link. There will be realized a CAD 3D modelling of this power link. 3D modelling is generalized and there were used the powers of Catia V5R20 software. Parameterized approach provides a high flexibility in the design, meaning that dimensional and shape changes of the semi-power link are very easy to perform just by changing some parameters. Several new versions are proposed for the anti-roll power link body. At the end of the work, it is made a static analysis of the semi-power link model used in the suspension of vehicles OPEL ASTRA G, ZAFIRA, MERIVA, and constructive optimization of its body.
\end{abstract}

Key words: CAD modelling, finite element methods, stabilizer bar, parameterized modelling, static analysis

\section{Introduction}

CAD modelling of auto parts is a research method that is useful when we study the behaviour of functional sub-assemblies of the vehicle.

Modern 3D design of auto parts gives first and foremost a very high flexibility in design due to possible parameterized approach by using dedicated software suites such as Catia or ProEngineer but it is also the underlying element to achieve some behaviour simulations of these milestones in actual operating conditions.

The type of anti-roll power link or semi-power link that we want to study is the one mounted on OPEL ASTRA G, ZAFIRA or MERIVA because these vehicles are commonly used in Romania, being quite affordable to a wide range of drivers. On the other hand, reliability analyses carried out show that anti-roll power link is vulnerable under operating conditions specific to our country.

\section{CAD modelling of anti-roll power link body}

Before proceeding to the actual modelling of the power link, it is appropriate to make some remarks on the construction and operation of this major auto part.

As regards the construction, we can distinguish three main parts, namely: body of power link and the two pivot sub-assemblies through which the anti-roll power link is assembled on the damper and respectively on the stabilizer bar of the vehicle (Figure 1).

Functionally, anti-roll power link is a component part of the vehicle suspension which has two basic functions, namely: reducing the leaning of the vehicle and adjusting the vehicle balance. On poor quality roads, the vehicle shall enter a rocking motion, and the motion depends on the construction of power link body or the material of which they are made. 
Therefore, we believe that it is fully justified to perform a thorough study of power link body, even if the parts wearing faster within this subassembly are the pivot parts on the ends. Also, the body of anti-roll power link has been less studied in specialized literature compared to pivot wear.

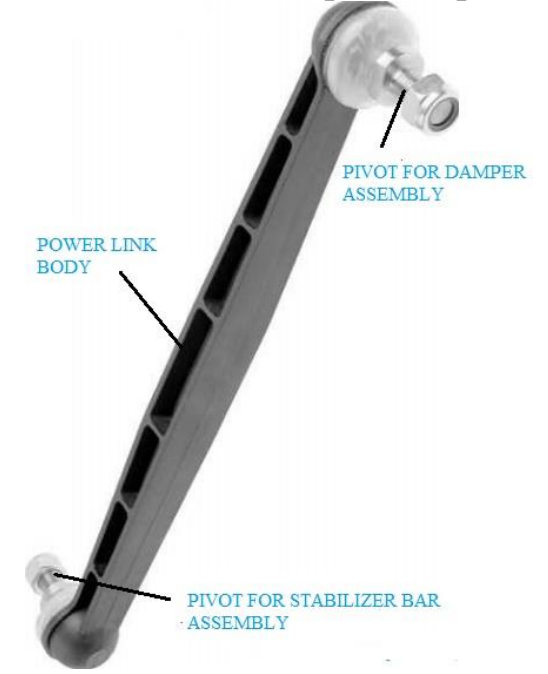

Figure 1: Anti-roll power link of OPEL ASTRA G, ZAFIRA, MERIVA

3D modelling of semi-power link will be carried out in the classical manner by using the software package CatiaV5R20.

For modelling, there were carried out successively as follows: modelling of semi-power link body, modelling of seats for mounting pivots to the ends, modelling of perimeter rib and finally of transverse ribs.

As a principle, modelling was done in a generalized and parametrized manner. Even if sometimes it requires a more difficult modelling, this has the great advantage of allowing many customizations, resulting in many constructive forms that can be further studied by other methods such as the method of finite element.

For 3D modelling of the full body of semi-power link it was considered the origin of of triorthogonal system of the body within its centre of symmetry, for the future use of symmetrisation functions allowing flexibility in modelling.

The next step is the realization of the beam shape of equal strength in $\mathrm{xOy}$ plane. To achieve other recesses will be used the facilities of Catia software to mirror the constructive entities. After this symmetrisation is created the basic shape of the anti-roll power link body.

Further is modelled the perimeter rib that results by creating a pocket entity on both parallel sides of the $\mathrm{yOz}$ plane of the body. To achieve the perimeter rib on the opposite side it will be applied again the Mirror function for entities, and the recess previously created will mirror against yOz plane.

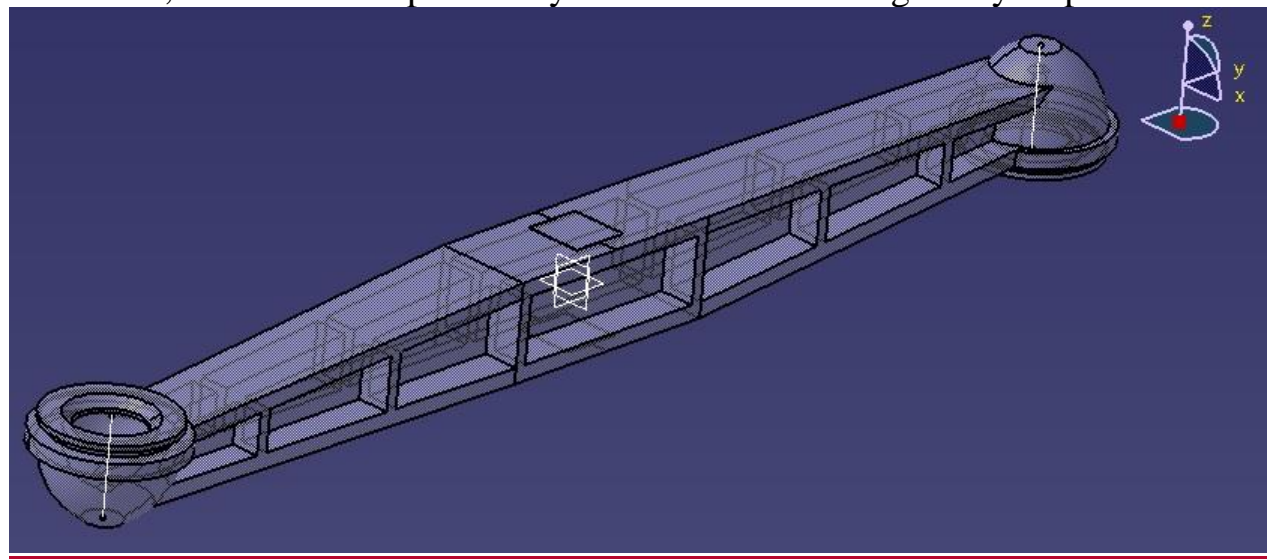

Figure 2: 3D anti-roll power link

We then proceed to modelling the ends of anti-roll power link, namely the seats for mounting pivots. Also for reasons of parameterization and subsequent flexibility, first will be modelled the outer surface of the seat. Using the same steps, will be realized the surface of inner revolution of the pivot seat. In terms of its constructive shape, the second seat is identical to the first, the only difference between them is its positioning towards the body of semi-power link. 
To complete the model, further will be modelled the transverse ribs. After realizing the ribs located on the other side of the body, in the same manner, modelling of semi-power link is completed, the final model is shown in figure 2 .

Since the model of the body of semi-power link was modelled after some parameters, by simply introducing new values to the parameters, it results customizations that may be object of further study.

Customizations proposed and models resulted are as follows:

- Changing the angle creating the beam shape of equal strength in xOy plane (figure 3);

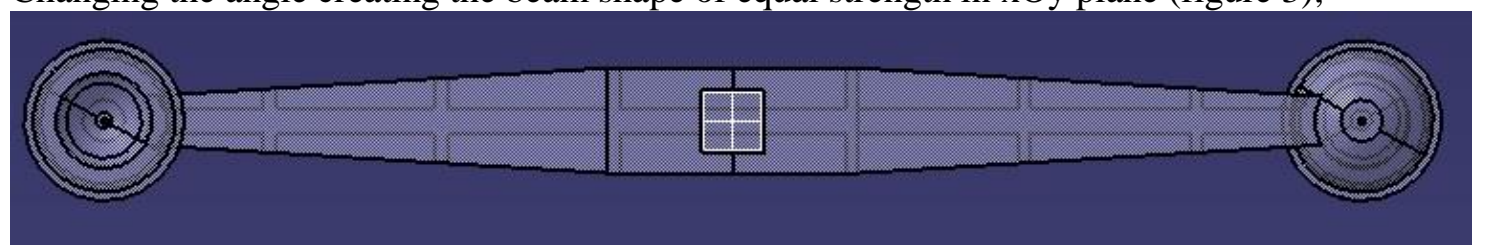

Figure 3. Constructive shapes with the angle of inclination of symmetrical surfaces changed in $x O y$ plane.

- $\quad$ Thinning or thickening of the middle area of the body in both $\mathrm{xOy}$ and $\mathrm{yOz}$ planes (figures 4, 5);

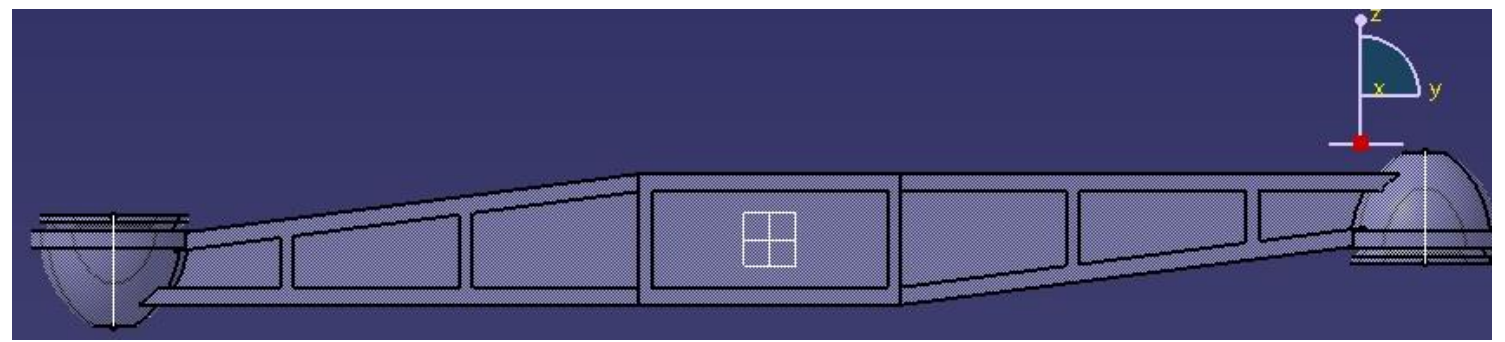

Figure 4. Thickened model in the middle area on zOy plane

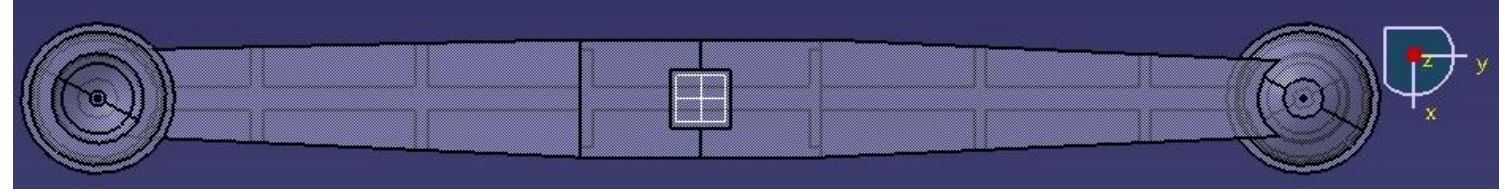

Figure 5. Thickened model in the middle area on $x O y$ plane

- $\quad$ Changing the inclination of body surface in $\mathrm{yOz}$ plane (figure 6);

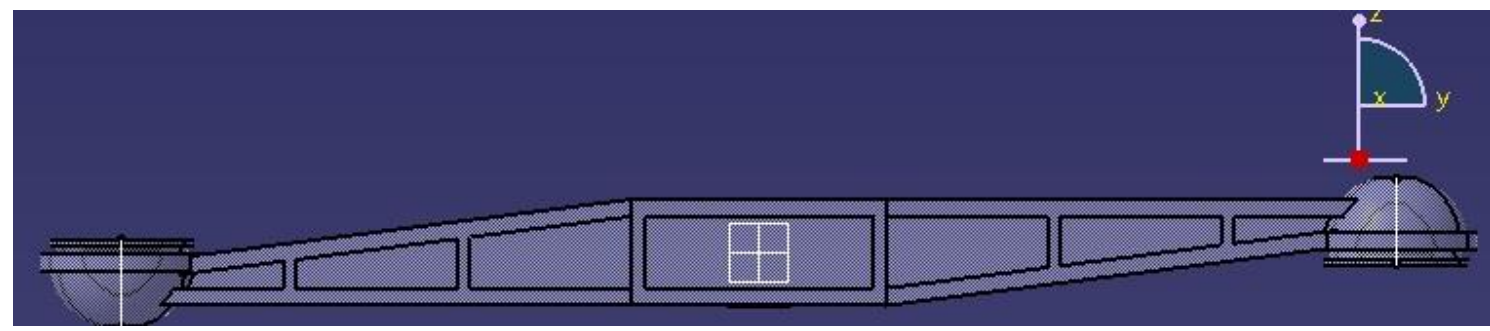

Figure 6. Model changed in terms of inclination of surfaces on zOy plane

- $\quad$ Thickening or thinning of perimeter rib (figure 7);

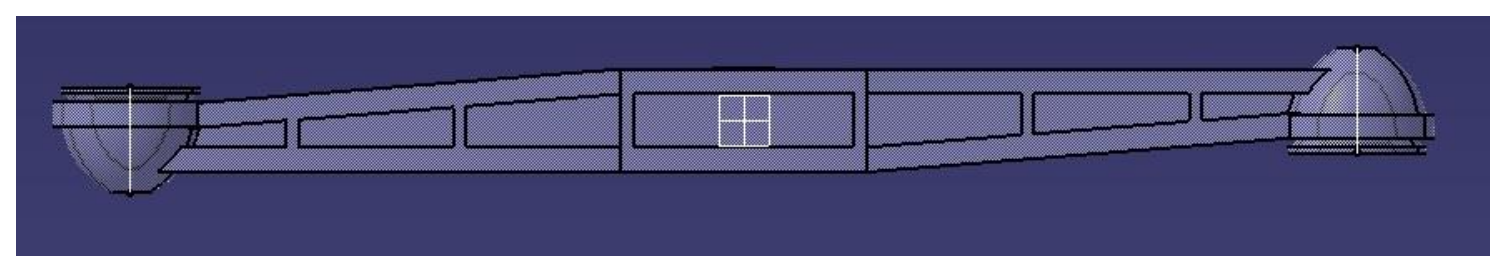

Figure 7. Model of thickened perimeter rib

- $\quad$ Changing the position of transverse ribs and of their sizes (figure 8)

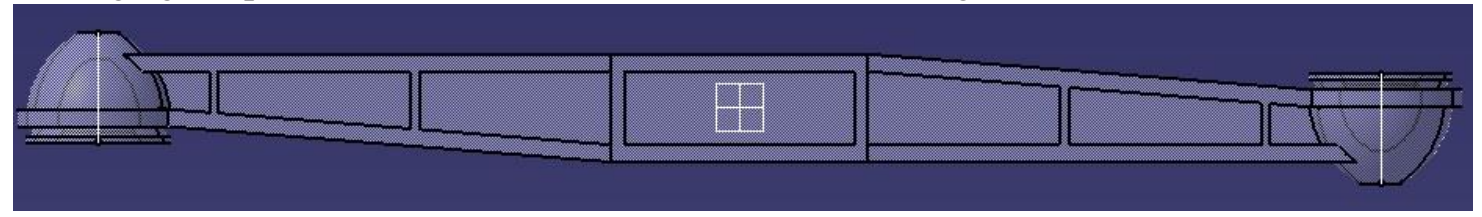

Figure 8. Model of thinned transverse rib and the increased distance between the ribs

As already seen, parameterized modelling enables a large enough study on constructive shape of anti-roll power link. 
Starting from the modelling presented in detail through constructive adjustments, there are several types of anti-roll power link bodies that can be suggested.

So, if the idea of ribs modelling is abandoned, will result a semi-power link of whose body is full. Another version is featured by the absence of transverse ribs, the body having only the perimeter rib. Another body model can be modelled without transverse ribs, by replacing them with a longitudinal rib passing through the centre of symmetry of the body and medially including xOy plane.

Finally, the last suggestion is a model of high rigidity, being basically a combination between the original model with transverse ribs and the model with longitudinal rib. The model is shown in figure 9.

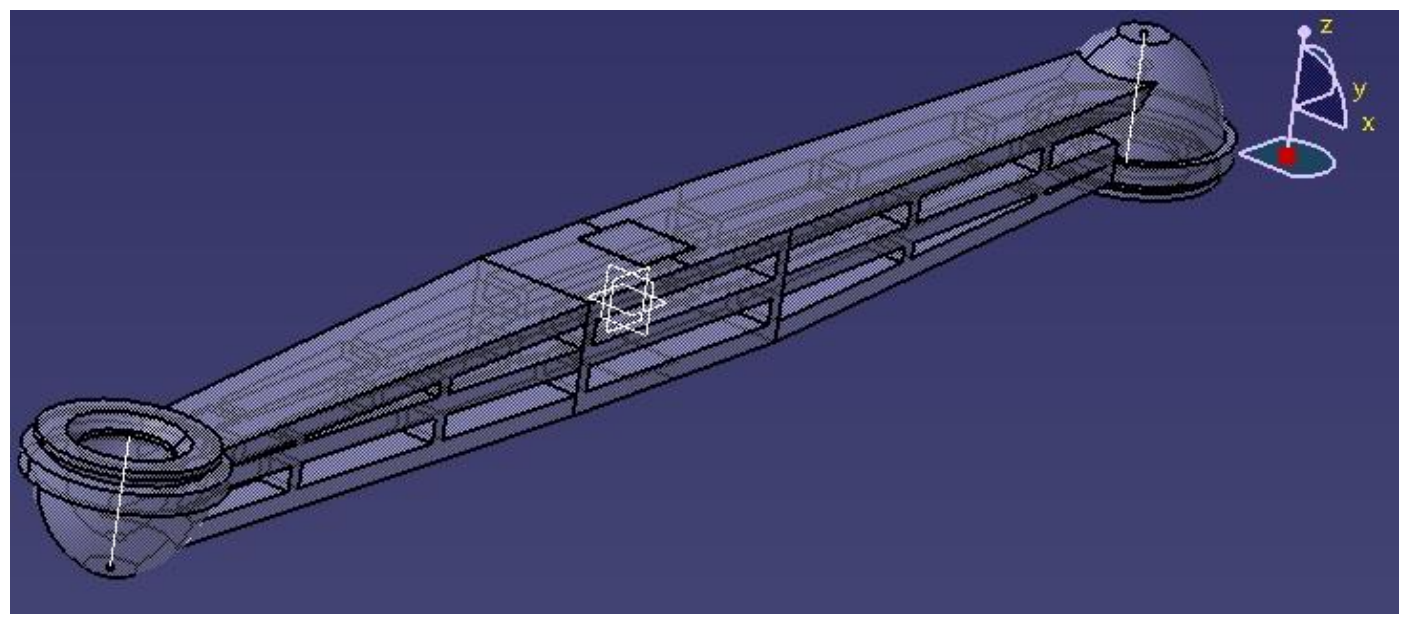

Figure 4.7.9. Semi-power link body with longitudinal rib and transverse ribs

The generalized design shown above is a starting point for constructive and functional optimization of the anti-roll power link body.

\section{Static analysis applied to anti-roll power link}

After saving the model previously realized in .stp format, it was introduced in the analysis software and was discretized.

One of the most important modules of Ansys software is the structural one that, due to its broad facilities, was selected for the study of static behaviour of anti-roll power link.

For the discretization of spatial structure of anti-roll power link, there were used Solid 92 finite elements.

The geometric model initially discretized of the power link is shown in figure 10. Material data introduced for the static analysis were as follows: elastic modulus (Young's modulus) $\mathrm{E}=7200 \mathrm{MPa}$ and coefficient of transverse contraction $v=0.35$.

Figure 11 shows how they have been applied the loads and constraints on the power link. Thus, in the support areas with such damper, were applied with the stabilizer bar 'remote displacement' constraints, cancelling translations on axes Ox and Oy and leaving free the translation on Oz.

As regards rotations, were cancelled the two rotations around axes Ox and Oy and was left free the rotation around axis Oz. the applied load was a moment whose total value was $\mathrm{M}=22500 \mathrm{Nmm}$, resulted frm calculation. 


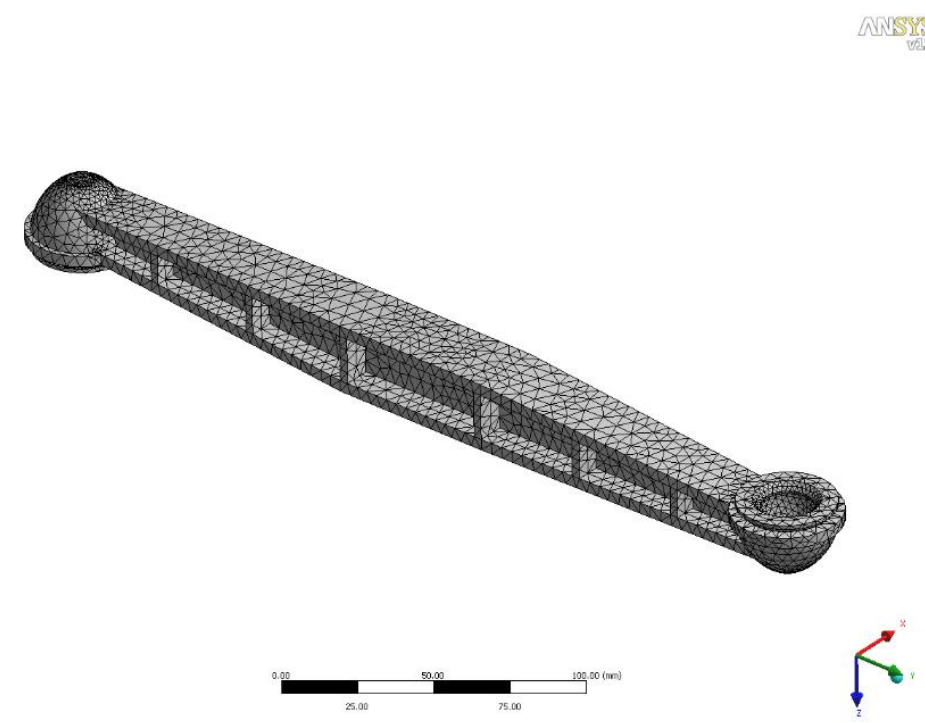

Figure 10. Discretized geometric model of anti-roll power link

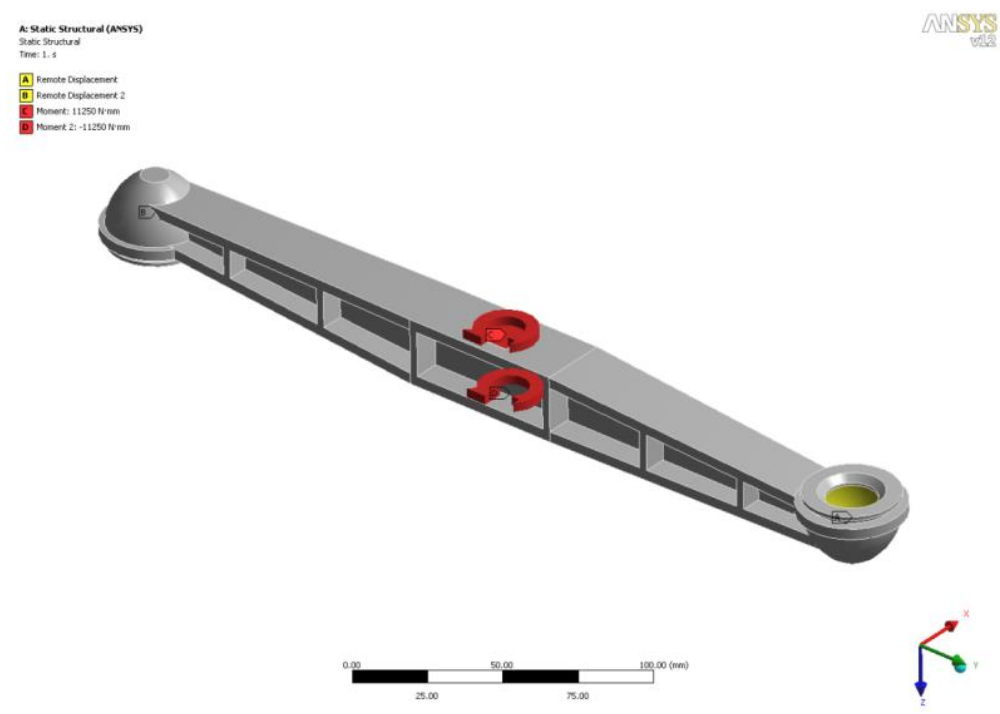

Figure 11. Loading mode of anti-roll power link during static analysis

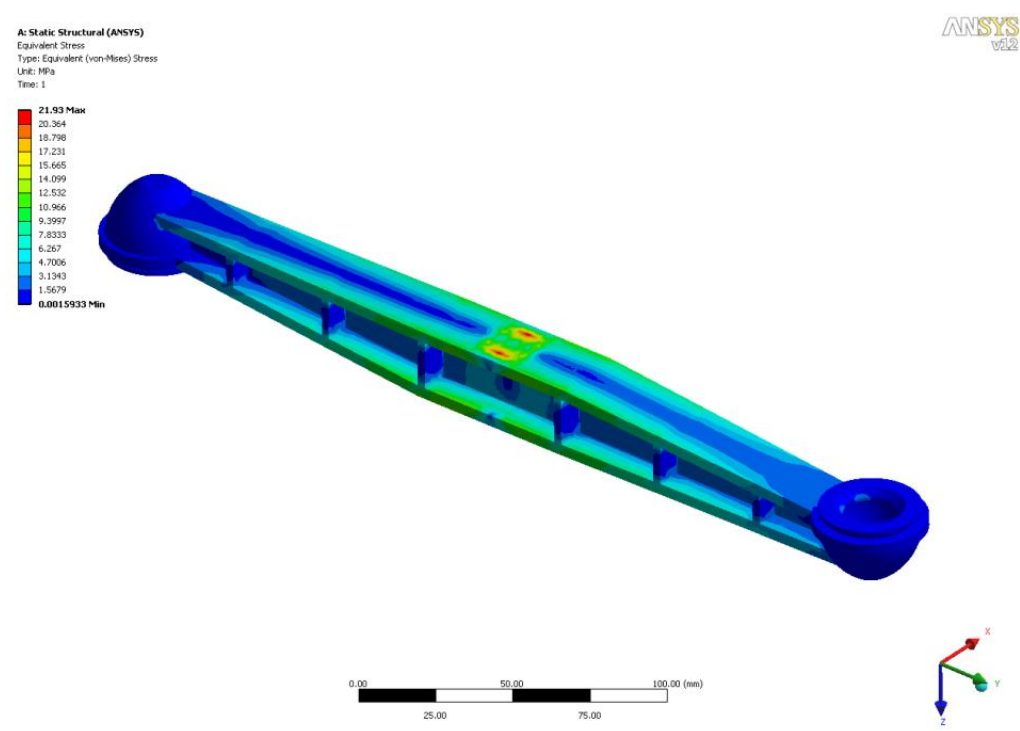

Figure 12. Variation of Von Mises equivalent strain $\sigma \mathrm{VM}$ [MPa] 


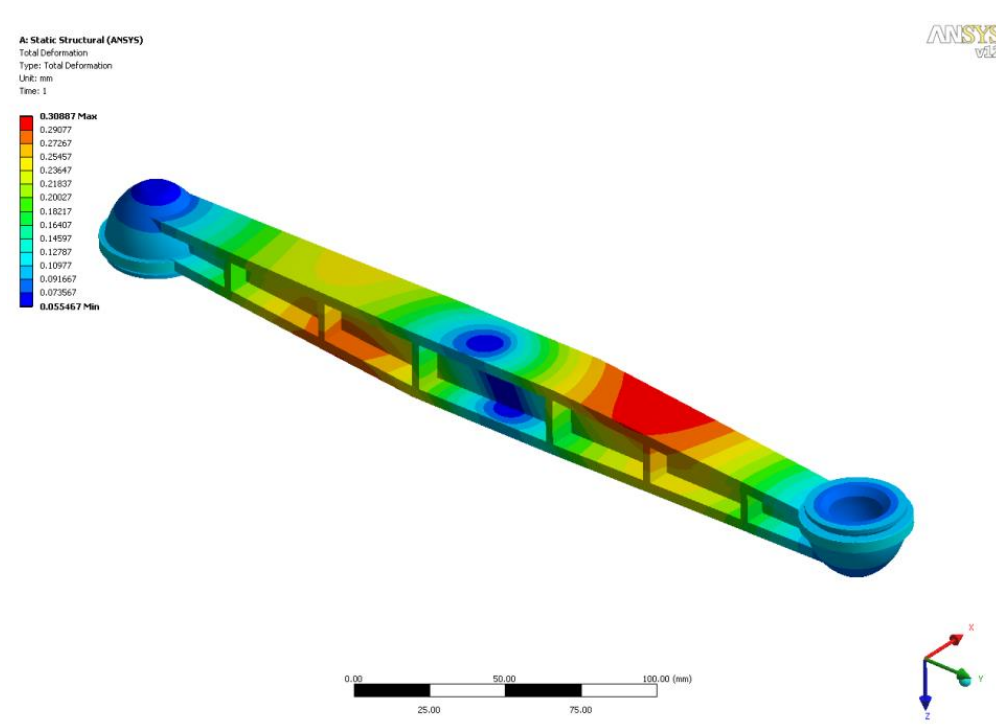

Figure 13 Variation of nodal displacements utot [mm]

After the static analysis, were obtained the values of main stresses, of Von Mises equivalent stress (Fig. 12), the value of total displacement (Fig. 13), and the safety factor in static analysis (Fig. 7.3.5).

The maximum values of main stresses are $\sigma 1=13.23 \mathrm{MPa}, \sigma 2=3.41 \mathrm{MPa}, \sigma 3=12.76 \mathrm{MPa}$. By combining these stresses, it results for Von Mises equivalent stress a value of $\sigma \mathrm{VM}=21.93 \mathrm{MPa}$, lower than the admissible material resistance for PA66-GF of $130 \mathrm{MPa}$. Therefore, it is obtained a value of 5.92 for the safety factor. The maximum nodal displacement is utot $=0.31 \mathrm{~mm}$.

\section{Conclusions}

We can conclude that parameterized modelling of anti-roll power link offers good flexibility in its designing and optimizing. One can easily modify the entities forming the power link body and can model new constructive versions.

After running numerical analyses by finite elements method, we can draw the following conclusions: static load; using the finite element method revealed the state of stress and strains in anti-roll power link upon

- $\quad$ for the anti-roll power link studied, the maximum value of Von Mises equivalent stress does not exceed the admissible material resistance for PA66-GF, obtaining values between 5.82 and 6.23 for the safety factor in static analysis;

- $\quad$ the maximum value of nodal displacement for the three geometric models of anti-roll power links is between 0.31 and $0.53 \mathrm{~mm}$, which are acceptable for anti-roll power links;

- based on these results, it is possible to perform a topological optimization of the body of semi-power link bending

numerical researches can be validated by experimental studies in eccentric bending and pure

\section{References}

1. Roman, L.I. - CERCETĂRI PRIVIND OPTIMIZAREA SUSPENSIILOR AUTOTURISMELOR EXPLOATATE ÎN ROMÂNIA, Study report 4, Doctoral thesis, (2015)

2. Walter W. THIERHEIMER ş.a - Optimizarea mecanismelor pentru suspendarea şi rularea roţilor autovehiculelor. Editura Universităţii „TRANSILVANIA” Braşov 2008

3. Zienkiewicz O.C., The Finite Element Method, Vol. I and II, McGraw Hill, London, 1991.

4. $* * *$, ANSYS Release 12, User Guide, USA.

5. Pacoste C., Stoian V., Dubină D., Metode moderne în mecanica structurilor, Editura Ştiinţifică şi Enciclopedică, Bucharest, 1988. 